\title{
A Novel Hybrid Machine Learning Classification for the Detection of Bruxism Patients Using Physiological Signals
}

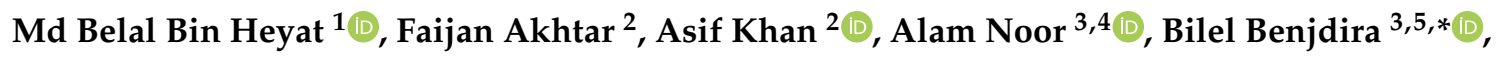 \\ Yumna Qamar ${ }^{6}$, Syed Jafar Abbas ${ }^{7}$ and Dakun Lai ${ }^{1, *(D)}$ \\ 1 School of Electronic Science and Engineering, University of Electronic Science and Technology of China, \\ Chengdu 610054, China; belalheyat@gmail.com \\ 2 School of Computer Science and Engineering, University of Electronic Science and Technology of China, \\ Chengdu 610054, China; faijanakhtar98@gmail.com (F.A.); asifkhan@uestc.edu.cn (A.K.) \\ 3 Robotics and Internet of Thing Lab, Prince Sultan University, Riyadh 11586, Saudi Arabia; \\ eng.alamnoor@gmail.com \\ 4 Department of Information and Communication Engineering, Harbin Institute of Technology, \\ Harbin 150001, China \\ 5 SEICTLab, LR18ES44, Enicarthage, University of Carthage, Tunis 2035, Tunisia \\ 6 Department of Orthodontics and Dentofacial Orthopedics, ZA Dental College and Hospital, \\ Aligarh Muslim University, Aligarh 202002, India; yumi.yumna@gmail.com \\ 7 School of Automation Engineering, University of Electronic Science and Technology of China, \\ Chengdu 611731, China; jafar1987abbas@rediffmail.com \\ * Correspondence: bbenjdira@psu.edu.sa (B.B.); dklai@uestc.edu.cn (D.L.)
}

Received: 13 September 2020; Accepted: 20 October 2020; Published: 22 October 2020

Featured Application: 1. The hybrid machine learning (HML) classifier can easily classify the subjects (healthy and bruxism), sleep stages (w and REM), and both with high accuracy. 2. The proposed system automatically detects the bruxism sleep disorder and sleep stages. 3. Single C4-A1 channel of the EEG signal found to be more accurate than ECG and EMG channels.

\begin{abstract}
Bruxism is a sleep disorder in which the patient clinches and gnashes their teeth. Bruxism detection using traditional methods is time-consuming, cumbersome, and expensive. Therefore, an automatic tool to detect this disorder will alleviate the doctor workload and give valuable help to patients. In this paper, we targeted this goal and designed an automatic method to detect bruxism from the physiological signals using a novel hybrid classifier. We began with data collection. Then, we performed the analysis of the physiological signals and the estimation of the power spectral density. After that, we designed the novel hybrid classifier to enable the detection of bruxism based on these data. The classification of the subjects into "healthy" or "bruxism" from the electroencephalogram channel (C4-A1) obtained a maximum specificity of $92 \%$ and an accuracy of $94 \%$. Besides, the classification of the sleep stages such as the wake (w) stage and rapid eye movement (REM) stage from the electrocardiogram channel (ECG1-ECG2) obtained a maximum specificity of $86 \%$ and an accuracy of $95 \%$. The combined bruxism classification and the sleep stages classification from the electroencephalogram channel (C4-P4) obtained a maximum specificity of $90 \%$ and an accuracy of $97 \%$. The results show that more accurate bruxism detection is achieved by exploiting the electroencephalogram signal (C4-P4). The present work can be applied for home monitoring systems for bruxism detection.
\end{abstract}

Keywords: machine learning; hybrid classifier; sleep disorder; dental disorder; EEG; ECG; EMG 


\section{Introduction}

Sleep is a vital need for human beings. It is characterized by altered consciousness, inhibited sensory movement, and reduced muscle movement [1]. It is found in all zoological species such as amphibians, animals, humans, insects, mammals, and reptiles. Some species sleep with open eyes, while most species sleep with closed eyes. Sleep stages are divided into four parts such as wake (w), light (non-rapid eye movement (NREM) 1 and 2), deep (NREM 3), and rapid eye movement (REM) [2-5].

Lack of sleep affects human life and causes many health problems: memory issues, mood changes, concentration issues, risk of diabetes, increased risk of heart diseases, weight gain, high blood pressure, and increased driver crash risk [6-9]. Lack of sleep also impacts negatively on a person's energy towards work, health, and emotional balance [10]. Good sleep is an indicator of a healthy person. It is a common phenomenon that minor sleep loss can decrease efficiency, energy levels, ability to handle stress, and mood [11]. Ignorance of sleep problems and sleep disorders is a serious issue because they may cause big damages like accidents, frustration, poor job performance, and stress [12]. The survey report of China sleep medicine and white paper on the internet (http://en.people.cn/n3/2018/0322/c90000-9440441.html, http://www.china.org.cn/china/2018-03/21/content_50731983.htm) in 2018 showed that 50 to 60 million Chinese people suffered from a sleep disorder [13]. Another survey shows that 3.5 million people in the UK and more than 70 million people from the US suffer from sleep disorders [14]. Another sleep survey (https://timesofindia.indiatimes.com/city/lucknow/World-Sleep-Day-93-Indians-are-sleepdeprived/articleshow/46547288.cms) shows that $93 \%$ of Indians suffer from poor sleep [15]. Sleep disorders are mainly classified as problems in staying awake, problems in falling asleep, and abnormal activity in sleep [16].

Bruxism is a type of sleep disorder in which people clench, chew, and grind their teeth. It is defined as a parafunctional habit consisting of involuntary rhythmic or spasmodic nonfunctional gnashing, grinding, or clenching of teeth [17]. This clenching leads to occlusal trauma, causing flattened, fractured, or chipped teeth (Figure 1). It is classified into two types such as awake bruxism and sleep bruxism. If the person grinds their teeth during awake time, it is called awake bruxism; otherwise, if the person grinds their teeth in their sleep, it is called sleep bruxism [18]. The symptoms of bruxism are grinding or clenching of teeth, flattened, fractured or tipped teeth, pain and sensitivity in teeth, abnormal jaw function, facial pain, earache, and sleep disturbances [19].

(A) Healthy Teeth

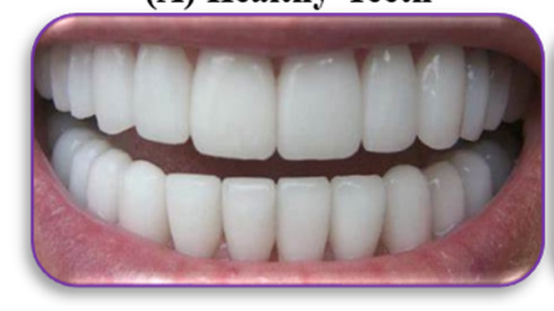

(B) Bruxism affected Teeth

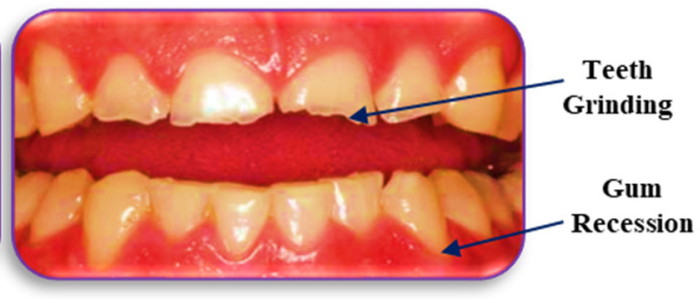

Figure 1. Comparison between (A) healthy and (B) bruxism teeth of the human. The grinding of the tooth is shown in the case of bruxism.

The diagnosis of bruxism is through various modalities, including history taking, clinical examination of teeth, intraoral appliances to observe facets, and bite force recording. A definitive diagnosis of bruxism is an expensive process that requires conducting a sleep study in an organized environment, usually at sleep clinics, to measure multiple factors and physiological indications during sleep. Some systems such as electrocardiogram (ECG) (cardiac signal) [20,21], electrooculogram (EOG) (eye signal), electroencephalogram (EEG) (brain signal) [22], and electromyogram (EMG) (muscles signal) [23] are used in automatic sleep stage detection [24]. Guillot et al. [25] evaluated the clinical practice on French dental clinicians. The authors used one thousand three hundred and eighty-eight practitioners based on five methods, such as oral rehabilitation, treatment of the patient with occlusal splints, sociodemographic characteristics, diagnosis, and management of sleep 
bruxism. The work discovered $16.8 \%$ wide inequality and inadequate diagnosis, and $21.9 \%$ of applicants planned cognitive-behavioral treatment. Saczuk et al. [26] evaluated the screening and detection method of bruxism. The researchers used the total number of sixty adults, including twenty-five healthy and thirty-five with bruxism. They evaluated the masseter muscle activity of the body. Maeda et al. [27] studied the validity of the detection of bruxism using EMG and a cut-off rate with optimum sympathy and specificity. A total number of 16 subjects were used in this work. The authors suggested that one-channel EMG and a cut-off value are appropriate for the detection of bruxism disorder. Miettinen et al. [28] suggested that the ambulatory electrode is the highest-precision device for the diagnosis of bruxism sleep disorder and sleep stage scoring. Ruhland et al. [29] represented the diagnosis of sleep bruxism using analysis and acquisition of the human masseter muscle by an EMG signal. Martinez et al. [30] investigated that remote communication and piezoelectric sensors are used in the diagnosis of bruxism sleep disorder. Kostka and Tkacz [31] investigated that multi-source data with sympathovagal balance valuation are used for the early diagnosis of bruxism disorder stages. Jirakittayakom and Wongsawat [32] designed an EMG instrument for the detection of sleep bruxism patients on the masseter muscle.

We propose a new detection system for bruxism using the extraction of power spectral density on physiological sleep recordings such as ECG (ECG1-ECG2), EMG (EMG1-EMG2), and EEG (C4-P4 and C4-A1). Initially, the physiological signal is extracted from the sleep database. The 10/20 standard sleep recording system recorded these data. It is used in the research work of sleep disorders such as circadian rhythm sleep disorders, insomnia [33-37], bruxism [38-40], sleep apnea, restless leg syndrome, narcolepsy [41], and nocturnal frontal lobe epilepsy [42]. We preprocessed the signals using a low-pass finite impulse response (FIR) filter to remove the noise of the signal. After filtering the signal, we estimated the power spectral density of the signals. Finally, we classified the sleep stages ( $w$ and REM) [43,44], subjects (bruxism and healthy), and combined subjects (bruxism and healthy) and sleep stages ( $w$ and REM) from the novel hybrid machine learning (HML) classifier. The proposed hybrid classifier is the combination of ten machine learning classifiers, namely K-nearest neighbor $(\mathrm{KNN})$, support vector machine (SVM), random forest (RF), naive Bayes (NB), linear regression (LR), classification and regression tree (CART), linear discriminant analysis (LDA), AdaBoost (AB), gradient boosting (GB), and extra trees (ET). There are two common issues in the previous research of bruxism. Firstly, there is a large number of features used in the studies, which results in a high computational load. Secondly, most of these methods did not represent a classification with sleep stages. We address these challenges in this study. Further, we focus on some goals, including estimation of the power spectral density, applying the novel HML classifier for the classification methods on the same feature, and comparison between the HML classifier and different previously used classifiers. The proposed study is organized as follows: the used database is presented in Section 2. The feature extraction and novel classification methods are presented in Section 3. The evaluation of the results and related discussion are presented in Section 4. Finally, the conclusions of this paper are presented in Section 5.

\section{Materials}

Bruxism and healthy individuals were collected from the physionet website, which offers free access to the collection of recorded healthy and patient data [45]. The signals of this database included EEG, ECG, EOG, EMG, and respiration [46]. The sleep monitoring system recorded bilateral EOG, six EEG channels, two channels of right- and left-leg EMG, respectively, submental EMG, nasal respiration thermistor, and ECG channel. The sampling rates of the data were $200 \mathrm{~Hz}$.

In this proposed work, we used EEG, ECG, and EMG channels such as C4-P4, C4-A1, ECG1-ECG2, and EMG1-EMG2 for the detection of bruxism. Further, we used 936 segments including 244 of ECG1-ECG2, 244 of EMG1-EMG2, 224 of C4-P4, and 224 of C4-A1 from $8.5 \pm 0.577$ (mean \pm SD) subjects in the proposed work shown in Table 1. The duration of one segment of the signal is $60 \mathrm{~s}$. All channels have two sleep stages, such as $\mathrm{w}$ and REM. The total duration of the $56,160 \mathrm{~s}$ segments is used in the proposed work. 
Table 1. Dataset of the present work.

\begin{tabular}{|c|c|c|c|c|c|c|c|c|}
\hline \multirow{2}{*}{$\begin{array}{l}\text { Name of the } \\
\text { Physiological } \\
\text { Signal }\end{array}$} & \multirow{2}{*}{$\begin{array}{c}\text { Channel of the } \\
\text { Physiological } \\
\text { Signal }\end{array}$} & \multicolumn{3}{|c|}{$\begin{array}{l}\text { Number of the Subjects } \\
\text { (n) }\end{array}$} & \multicolumn{3}{|c|}{$\begin{array}{l}\text { Number of the Segment } \\
\text { (n) }\end{array}$} & \multirow{2}{*}{$\begin{array}{c}\begin{array}{c}\text { Duration of } \\
\text { the Signal }\end{array} \\
\text { (s) }\end{array}$} \\
\hline & & Male & Female & Total & Bruxism & Healthy & Total & \\
\hline ECG & ECG1-ECG2 & 6 & 3 & 9 & 149 & 95 & 244 & 14,640 \\
\hline EMG & EMG1-EMG2 & 6 & 3 & 9 & 149 & 95 & 244 & 14,640 \\
\hline EEG & C4-P4 & 4 & 4 & 8 & 140 & 84 & 224 & 13,440 \\
\hline EEG & C4-A1 & 4 & 4 & 8 & 140 & 84 & 224 & 13,440 \\
\hline \multirow{2}{*}{\multicolumn{2}{|c|}{$\begin{array}{l}\text { Mean } \\
\pm S D\end{array}$}} & 5 & 3.5 & 8.5 & 144.5 & 89.5 & 234 & 14,040 \\
\hline & & 1.154 & 0.577 & 0.577 & 5.196 & 6.350 & 11.547 & 692.820 \\
\hline
\end{tabular}

\section{Methods}

In this section, we will describe the techniques used for the detection of bruxism. For the sleep disorder, we collected, first, the dataset from the sleep database. After that, we extracted the physiological signals such as ECG (ECG1-ECG2), EMG (EMG1-EMG2), and EEG (C4-P4 and C4-A1). We preprocessed the channels and made the feature extraction of these channels. Then, the feature values were normalized before passing them to the novel HML classifier. The procedure is illustrated in Figure 2. The classification of subjects into "bruxism" and "healthy", the classification of sleep stages into " $w$ " and "REM", and the combined classification (both subjects and sleep stages) were performed by the novel HML classifier.

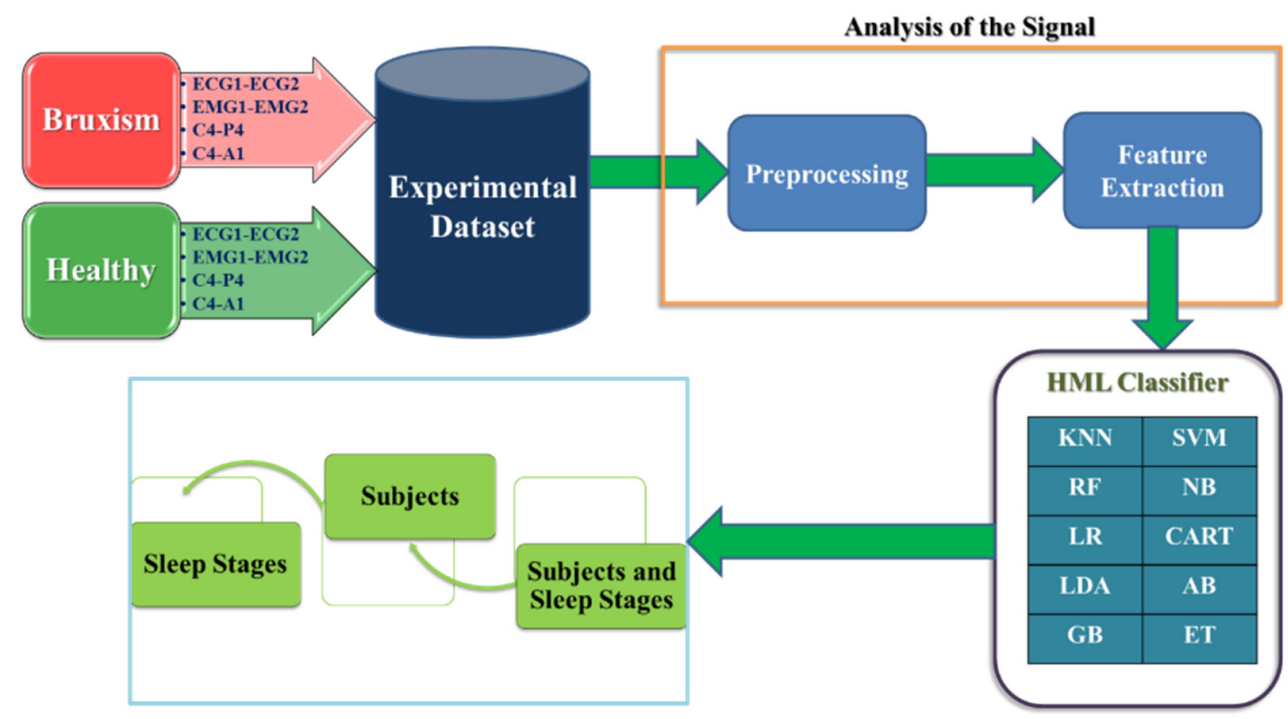

Figure 2. Organizational structure of the proposed work.

\subsection{Power Spectral Density}

We estimated the power spectral density using the Welch method. Peter D Welch discovered this method in 1967 [47]. It converts time series into (overlapping) segment data, calculating a modified periodogram of every segment, and takes the average of the power spectral density [48]. The average altered periodogram tends to reduce the variance [49]. Further, it estimates the relation to a single periodogram of aggregate data. Power spectral density offers signal power with respect to the frequency spectrum. We require specifying the number of frequency slots to allocate the power spectral that is called the number of fast Fourier transform [50,51]. The Welch techniques are described in Equations (1)-(3) below:

$$
\begin{gathered}
U=\frac{1}{L} \sum_{n=0}^{L-1}\left\{w_{h m}(n)\right\}^{2} \\
P_{w}(f)=\frac{1}{L U} \sum_{n=0}^{L-1}\left\{w_{h m}(n) x(n+i D) e^{-j 2 \pi f n}\right\}^{2}
\end{gathered}
$$




$$
P_{w}(f)=\gamma \sum_{n=0}^{L-1}\left(\left\{X_{a}^{n}\right\}^{2}+\left\{X_{b}^{n}\right\}^{2}\right)
$$

where $U$ is equal to compensate for the loss of signal, $L$ and $D$ are the data of the segment, $w_{h m}(n)$ is the hamming window, $\gamma$ is a constant, $X_{a}^{n}$ and $X_{b}^{n}$ are the real and imaginary part of the $n$th segment, and $P_{w}(f)$ is the Welch method.

\subsection{Hybrid Machine Learning (HML) Classifier}

The selection of a suitable classifier for efficient detection of bruxism is our goal in this study. However, the performance of a machine learning classifier varies from one problem to another. Hence, we opted to combine multiple machine learning classifiers to form a novel HML classifier. We combined ten classifiers including KNN, SVM, RF, NB, LR, CART, LDA, AB, GB, and ET. For implementation of the HML classifier, we used a scientific open source software, Anaconda [52,53]. We combined the machine learning output through majority voting in which we took the outputs of ten classifiers and checked the majority outputs. For example, if nine classifiers' output is bruxism patients but one classifier's output is healthy, then bruxism is the majority. Hence, our final output will be bruxism. The HML classifier improved the results and reduced the error of the system. In the literature, many classifiers based on hybridization of multiple machine learning classifiers were proposed for other problems. For example, Chen et al. [54] designed a hybrid AB classifier for the recognition of the cognitive radio network. Rawat et al. [55] designed a hybrid machine learning model using artificial neural network (ANN) and NB for the prediction of educational performance in the data mining field. Miskovic [56] suggested a hybrid model for the classification of decision support. Chen et al. [57] used the hybrid model for categorizing residential requests in natural language to provide timely replies back to citizens under the vision of digital administration services in smart cities. In these approaches, the designed HML models outperformed in all classifications.

\subsection{Evaluation of the Proposed System}

In this proposed work, we applied a novel 20-fold cross-validation model of the HML classifier to discriminate the bruxism and healthy subjects. It is evaluated with $234 \pm 11.547$ recording of the EMG1-EMG2, ECG1-ECG2, C4-P4, and C4-A1 channels, which includes $144.5 \pm 5.196$ bruxism and $89.5 \pm 6.350$ healthy recordings, with a time duration of $14.040 \pm 692.820 \mathrm{~s}$. The evaluations of the classification are applied in the EMG1-EMG2, ECG1-ECG2, C4-P4, and C4-A1 channels of the EMG, ECG, and EEG signals. Previously, we used these channels separately. However, in the proposed study, we used these four channels to find the best possible outcome of bruxism detection. It covered all important signals such as EMG, ECG, and EEG. We evaluated the final performance of the model by using different well-known parameters that are given in Equations (4)-(7).

$$
\begin{gathered}
\text { Sensitivity }=\left(\frac{T P}{(T P+F N)}\right) \\
\text { Specificity }=\left(\frac{T N}{(T N+F P)}\right) \\
\text { Accuracy }=\left(\frac{(T P+T N)}{(T P+T N+F P+F N)}\right) \\
M C C=\left(\frac{(T P \times T N)-(F P \times F N)}{\sqrt{(T P+F P)(T P+F N)(T N+F P)(T N+F N)}}\right)
\end{gathered}
$$

where $T P$ is the true positives, $F P$ is the false positives, $T N$ is the true negatives, and $F N$ is the false negatives. 


\section{Results and Discussion}

\subsection{Analysis of the Physiological Signals}

The physiological sleep channels, including ECG1-ECG2, EMG1-EMG2, C4-P4, and C4-A1 of the bruxism and healthy subjects, are shown in Figures 3 and 4 [19,40,48]. The 10/20 standard sleep recording system records these channels. Firstly, we extracted each channel and filtered each channel with a 200th order low-pass finite impulse response filter with a cut-off frequency of $25 \mathrm{~Hz}$ as noise removal. After filtration, we extracted the power spectral density of each physiological channel shown in Figures 5 and 6 . After normalizing the value of the power spectral density, we classified the signals in terms of subjects, sleep stages, and combined using the novel HML classifier.

(A) ECG1-ECG2 Channel of the Bruxism

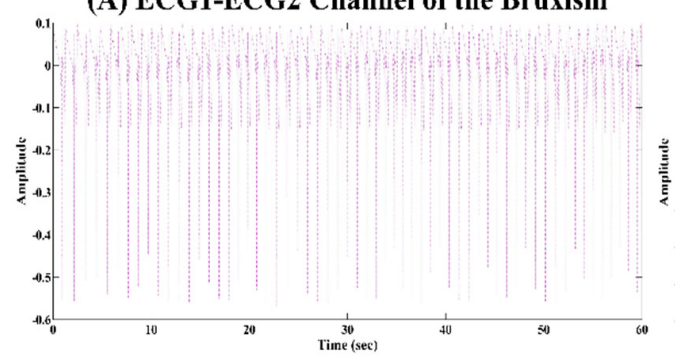

(C) C4-P4 Channel of the Bruxism

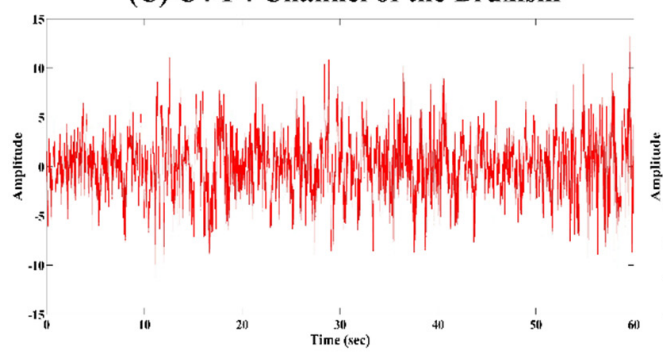

(B) EMG1-EMG2 Channel of the Bruxism

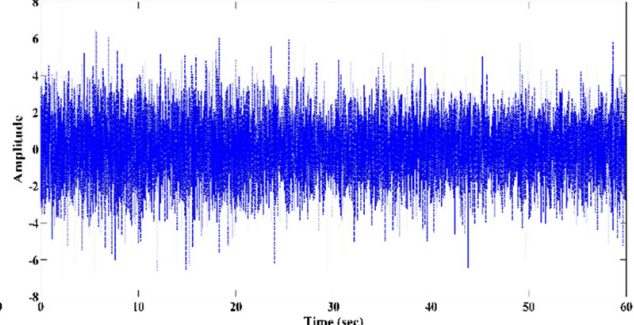

(D) C4-A1 Channel of the Bruxism

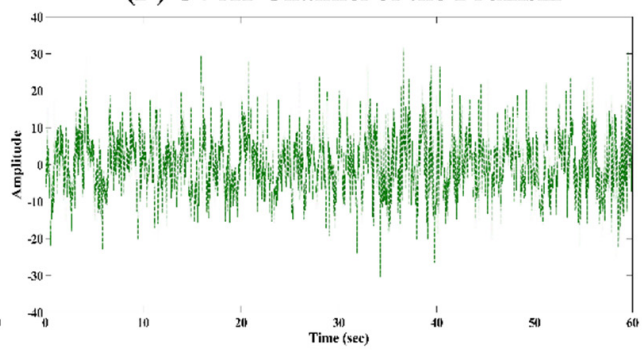

Figure 3. Signal representation of the sixty-second sleep physiological channels such as (A) ECG1-ECG2, (B) EMG1-EMG2, (C) C4-P4, and (D) C4-A1 of the bruxism subjects. A 10/20 standard sleep recording system recorded them.

(A) ECG1-ECG2 Channel of the Healthy

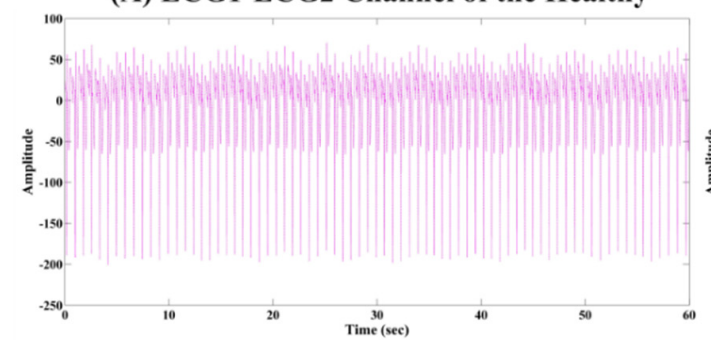

(C) C4-P4 Channel of the Healthy

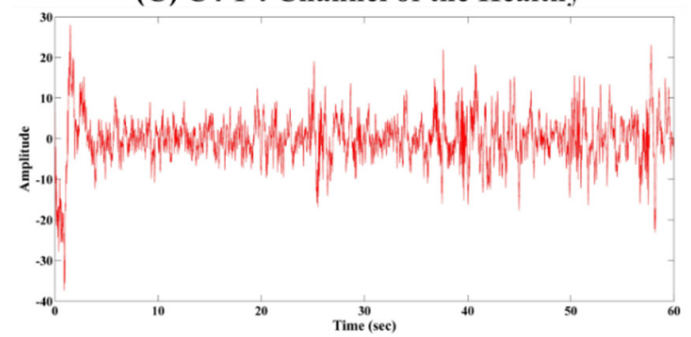

(B) EMG1-EMG2 Channel of the Healthy

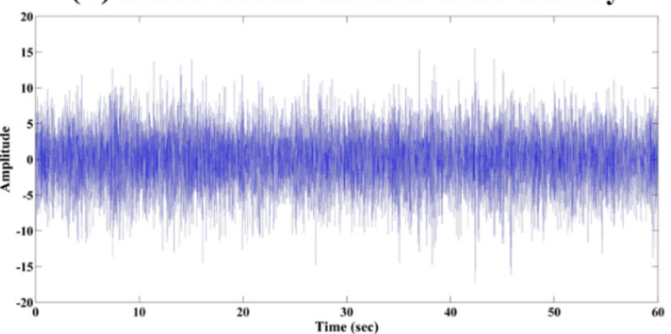

(D) C4-A1 Channel of the Healthy

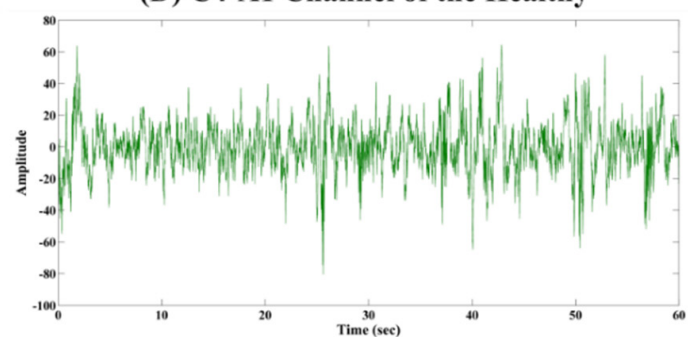

Figure 4. Signal representation of the sixty-second sleeps physiological channels such as (A) ECG1-ECG2, (B) EMG1-EMG2, (C) C4-P4, and (D) C4-A1 of the healthy subjects. A 10/20 standard sleep recording system recorded them. 

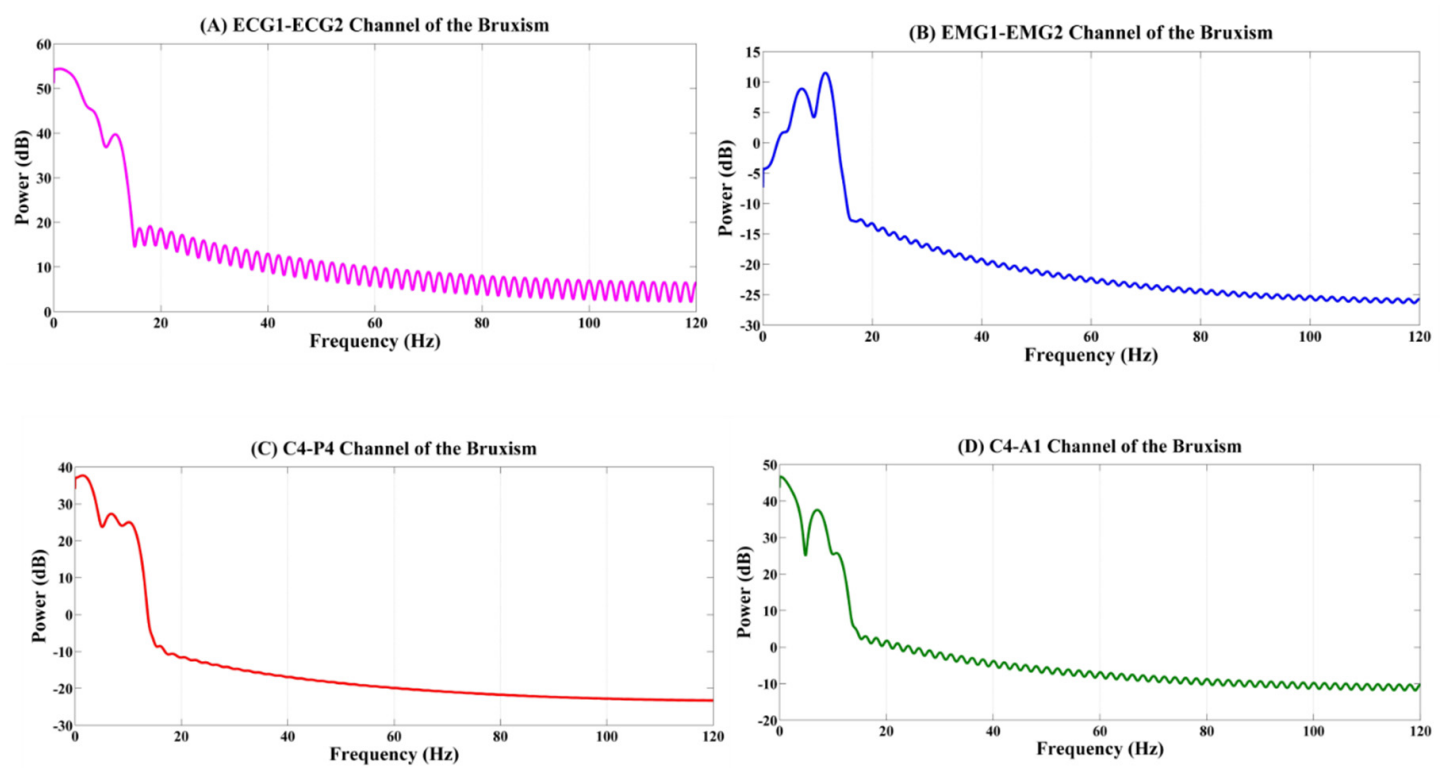

Figure 5. Power spectral analysis of the channels such as (A) the ECG1-ECG2 channel, (B) EMG1-EMG2 channel, (C) C4-P4 channel, and (D) C4-A1 channel of the bruxism subjects.
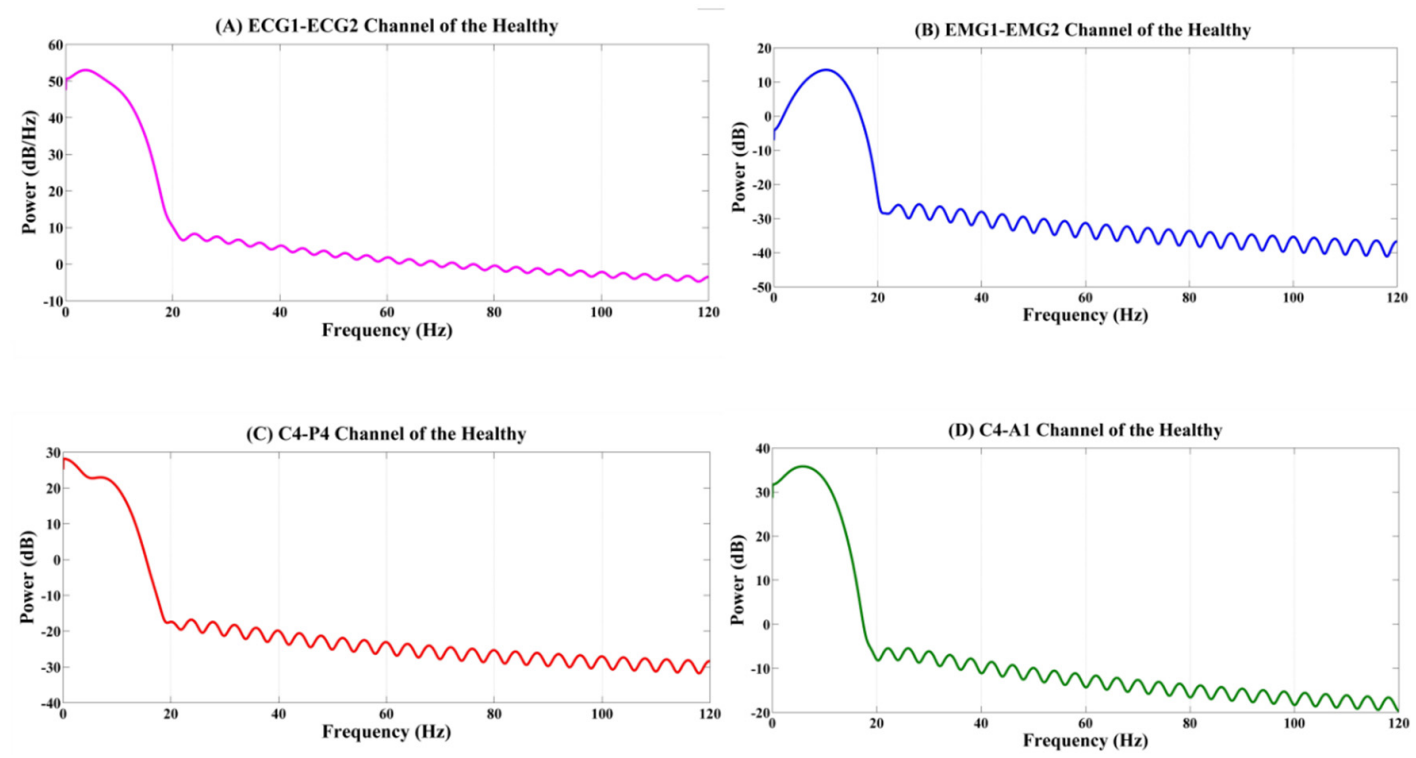

Figure 6. Power spectral analysis of the channels such as (A) the ECG1-ECG2 channel, (B) EMG1-EMG2 channel, (C) C4-P4 channel, and (D) C4-A1 channel of the healthy subjects.

\subsection{Classification Results Using Proposed Novel HML Classifier}

The performance for the classification of the subjects is shown in Table 2. Previously, we designed the diagnostic of bruxism sleep syndrome and sleep stages using a decision tree classifier with $81.25 \%$ accuracy [19]. Lai et al. [40] showed that the ECG channel and EMG channel are used for the detection of bruxism syndrome using power spectral density (PSD) techniques. They used the 488-min data for the two channels and classification of the subjects and stages using the machine learning method. Our proposed system, the C4-A1 channel, has the best performance in the subjects (bruxism and healthy) classification based on sensitivity, specificity, and accuracy, which are $95 \%, 92 \%$, and $94 \%$, respectively (Figure 7). 
Table 2. Performance of the subjects (bruxism and healthy) classification using the novel 20-fold cross-validation model of the HML classifier.

\begin{tabular}{cccccccccccc}
\hline Name of the Channel & PT & NT & TP & TN & FP & FN & F1 & MCC & Sen & Spe & Acc \\
\hline EMG1-EMG2 & 64 & 17 & 46 & 17 & 18 & 0 & 0.83 & 0.59 & 1 & 0.48 & 0.77 \\
ECG1-ECG2 & 78 & 3 & 46 & 3 & 32 & 0 & 0.74 & 0.22 & 1 & 0.08 & 0.60 \\
C4-P4 & 48 & 26 & 45 & 24 & 3 & 2 & 0.94 & 0.85 & 0.95 & 0.88 & 0.93 \\
C4-A1 & 47 & 27 & 45 & 25 & 2 & 2 & $\mathbf{0 . 9 5}$ & $\mathbf{0 . 8 8}$ & $\mathbf{0 . 9 5}$ & $\mathbf{0 . 9 2}$ & $\mathbf{0 . 9 4}$ \\
\hline Mean & 59.2 & 18.2 & 45.5 & 17.2 & 13.7 & 1 & 0.86 & 0.63 & 0.97 & 0.59 & 0.81 \\
\pm SD & 14.7 & 11.1 & 0.57 & 10.1 & 14.1 & 1.15 & 0.09 & 0.30 & 0.02 & 0.39 & 0.16 \\
\hline
\end{tabular}

PT: positive test; NT: negative test; TP: true positive; TN: true negative; FP: false positive; FN: false negative; MCC: Matthews correlation coefficient; Sen: sensitivity; Spe: specificity; Acc: accuracy. Bold is the best model.
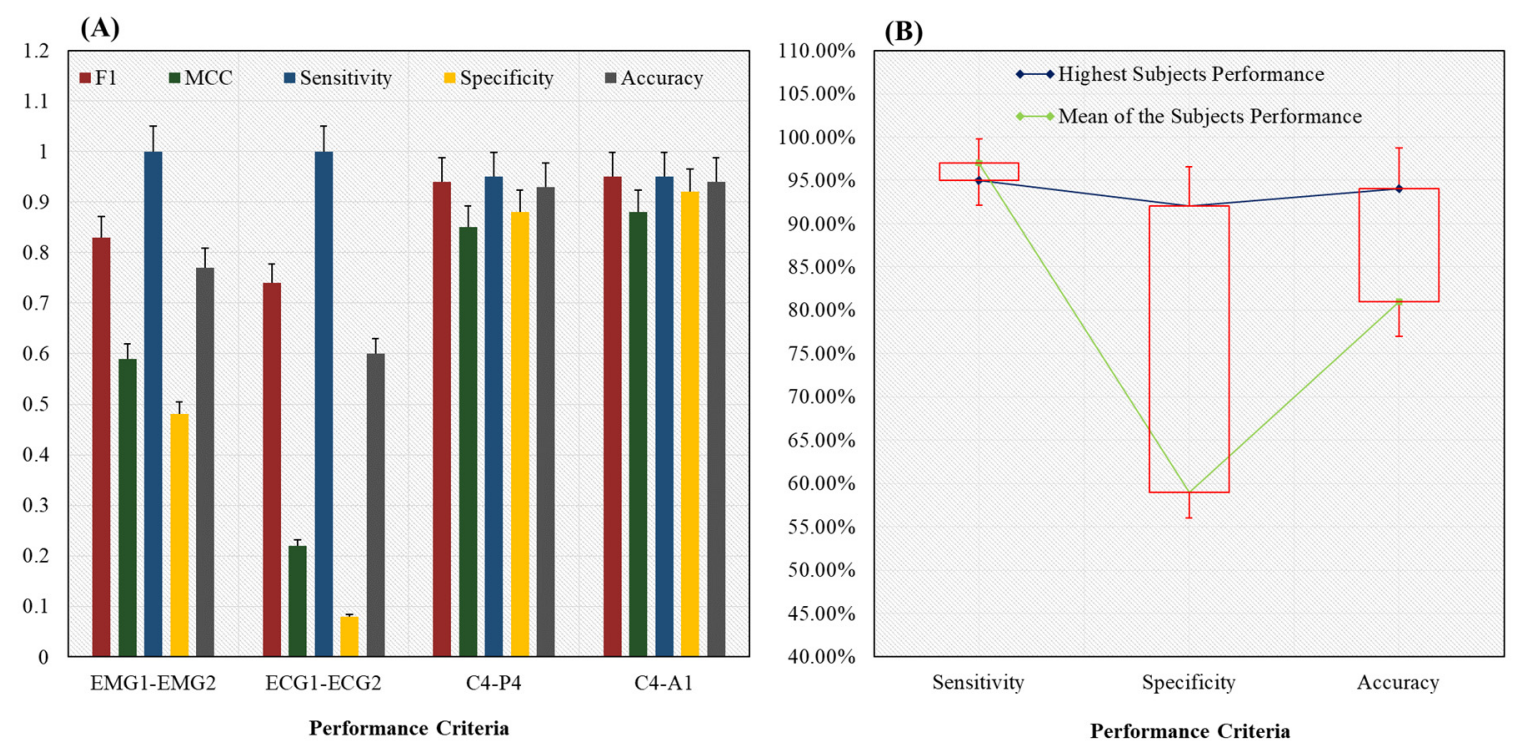

Figure 7. Performance analysis of the subjects (bruxism and healthy) classification using the novel 20-fold HML classifier. (A) Comparison between all channels such as EMG1-EMG2, ECG1, ECG2, C4-P4, and C4-A1 channels of the subjects classification. In addition, (B) highest and mean performance of the subjects classification. The C4-A1 channel of the EEG signal has the highest accuracy (94\%) in the subjects classification.

The performance of the sleep stages classification is shown in Table 3. Previously, the unsupervised learning architecture and hidden Markov model were used for the detection of sleep stages [58]. Boe et al. [59] used a multimodal sensor system evaluating hand acceleration, ECG, and ActiWatch for diagnosing sleep stages such as w, REM, and NREM. Bajaj et al. [60] designed an automatic system for diagnosing sleep stages using time-frequency images of the EEG signals. Matsuura et al. [61] studied that heart rate measurement is helpful and easy to use in sleep stage monitoring. They successfully calculated four stages and overall five stages with $66 \%$ accuracy of the system. Our proposed system, the ECG1-ECG2 channel, has the best performance in the sleep stages ( $w$ and REM) classification based on sensitivity, specificity, and accuracy, which are 100, 86, and 95\%, respectively (Figure 8). 
Table 3. Performance of the sleep stages ( $w$ and REM) classification using the novel 20 -fold cross-validation model of the HML classifier.

\begin{tabular}{cccccccccccc}
\hline Name of the Channel & PT & NT & TP & TN & FP & FN & F1 & MCC & Sen & Spe & Acc \\
\hline EMG1-EMG2 & 54 & 27 & 50 & 25 & 4 & 2 & 0.94 & 0.83 & 0.96 & 0.86 & 0.92 \\
ECG1-ECG2 & 56 & 25 & 52 & 25 & 4 & 0 & $\mathbf{0 . 9 6}$ & $\mathbf{0 . 8 9}$ & $\mathbf{1}$ & $\mathbf{0 . 8 6}$ & $\mathbf{0 . 9 5}$ \\
C4-P4 & 47 & 27 & 42 & 23 & 5 & 4 & 0.9 & 0.73 & 0.91 & 0.82 & 0.87 \\
C4-A1 & 59 & 15 & 45 & 14 & 14 & 1 & 0.85 & 0.57 & 0.97 & 0.5 & 0.79 \\
\hline Mean & 54 & 23.5 & 47.25 & 21.75 & 6.75 & 1.75 & 0.91 & 0.75 & 0.97 & 0.76 & 0.88 \\
ISD & 5.09 & 5.74 & 4.57 & 5.25 & 4.85 & 1.70 & 0.04 & 0.13 & 0.03 & 0.17 & 0.06 \\
\hline
\end{tabular}

Bold is the best model.
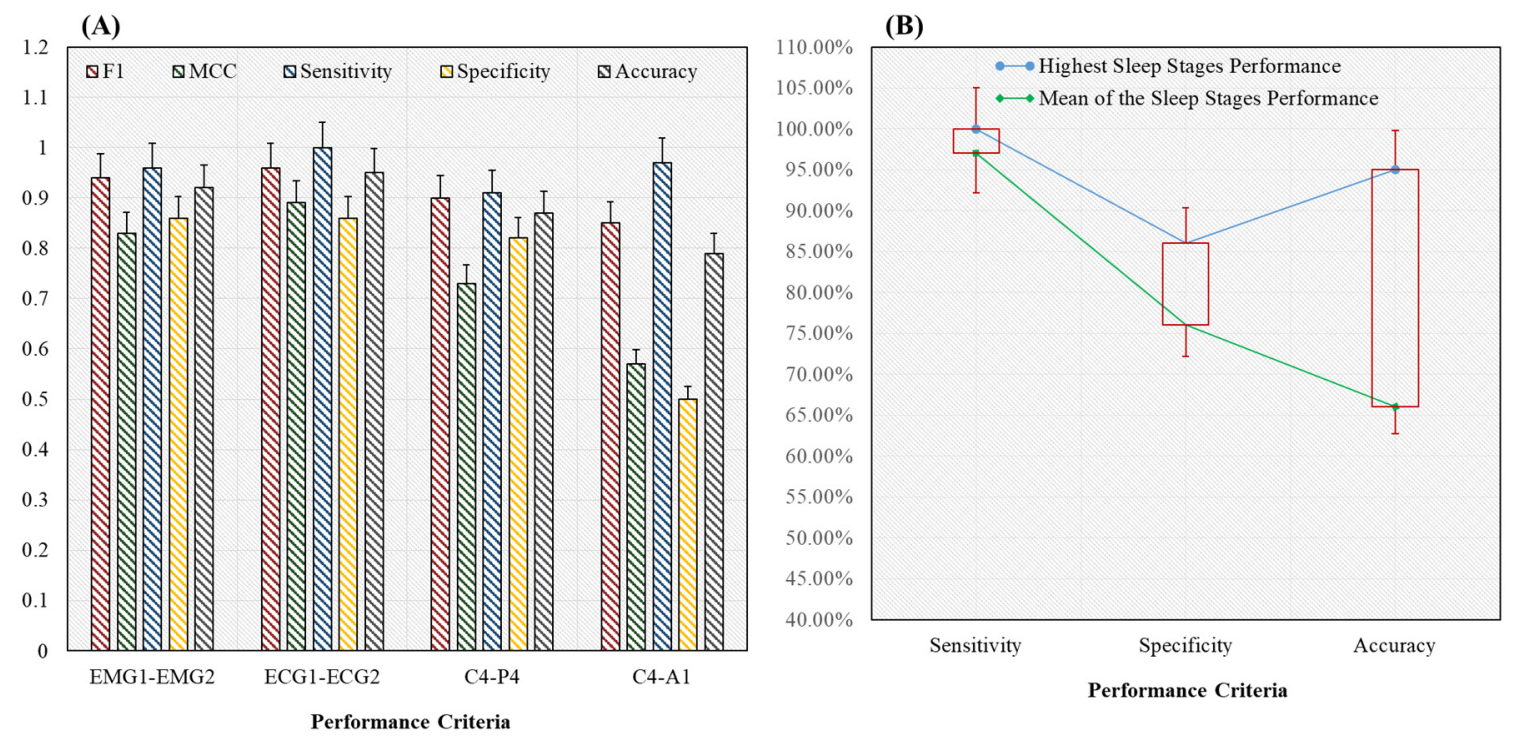

Figure 8. Performance analysis of the sleep stages ( $w$ and REM) classification using the novel 20-fold HML classifier. (A) Comparison between all channels such as EMG1-EMG2, ECG1, ECG2, C4-P4, and C4-A1 channels of the sleep stages classification. In addition, (B) highest and mean performance of the sleep stages classification. The ECG1-ECG2 channel of the ECG signal has the highest accuracy (95\%) in the sleep stages classification.

The performances for the combination of subjects and sleep stages classification are shown in Table 4. Our proposed system, the C4-P4 channel, has the best performance in the combined (subjects and sleep stages) classification based on sensitivity, specificity, and accuracy, which are 98, 90, and 97\%, respectively (Figure 9).

Table 4. Performance for the combination of subjects (bruxism and healthy) and sleep stages ( $\mathrm{w}$ and REM) classification using the novel 20-fold cross-validation model of the HML classifier.

\begin{tabular}{cccccccccccc}
\hline Name of the Channel & PT & NT & TP & TN & FP & FN & F1 & MCC & Sen & Spe & Acc \\
\hline EMG1-EMG2 & 63 & 18 & 63 & 17 & 0 & 1 & 0.99 & 0.96 & 0.98 & 1.00 & 0.98 \\
ECG1-ECG2 & 65 & 16 & 63 & 15 & 2 & 1 & 0.97 & 0.88 & 0.98 & 0.88 & 0.96 \\
C4-P4 & 63 & 11 & 62 & 10 & 1 & 1 & $\mathbf{0 . 9 8}$ & $\mathbf{0 . 8 9}$ & $\mathbf{0 . 9 8}$ & $\mathbf{0 . 9 0}$ & $\mathbf{0 . 9 7}$ \\
C4-A1 & 65 & 9 & 62 & 8 & 3 & 1 & 0.96 & 0.77 & 0.98 & 0.72 & 0.94 \\
\hline Mean & 64 & 13.5 & 62.5 & 12.5 & 1.5 & 1 & 0.97 & 0.87 & 0.98 & 0.65 & 0.96 \\
ISD & 1.15 & 4.20 & 0.57 & 4.20 & 1.29 & 0 & 0.01 & 0.07 & 0 & 0.37 & 0.01 \\
\hline
\end{tabular}



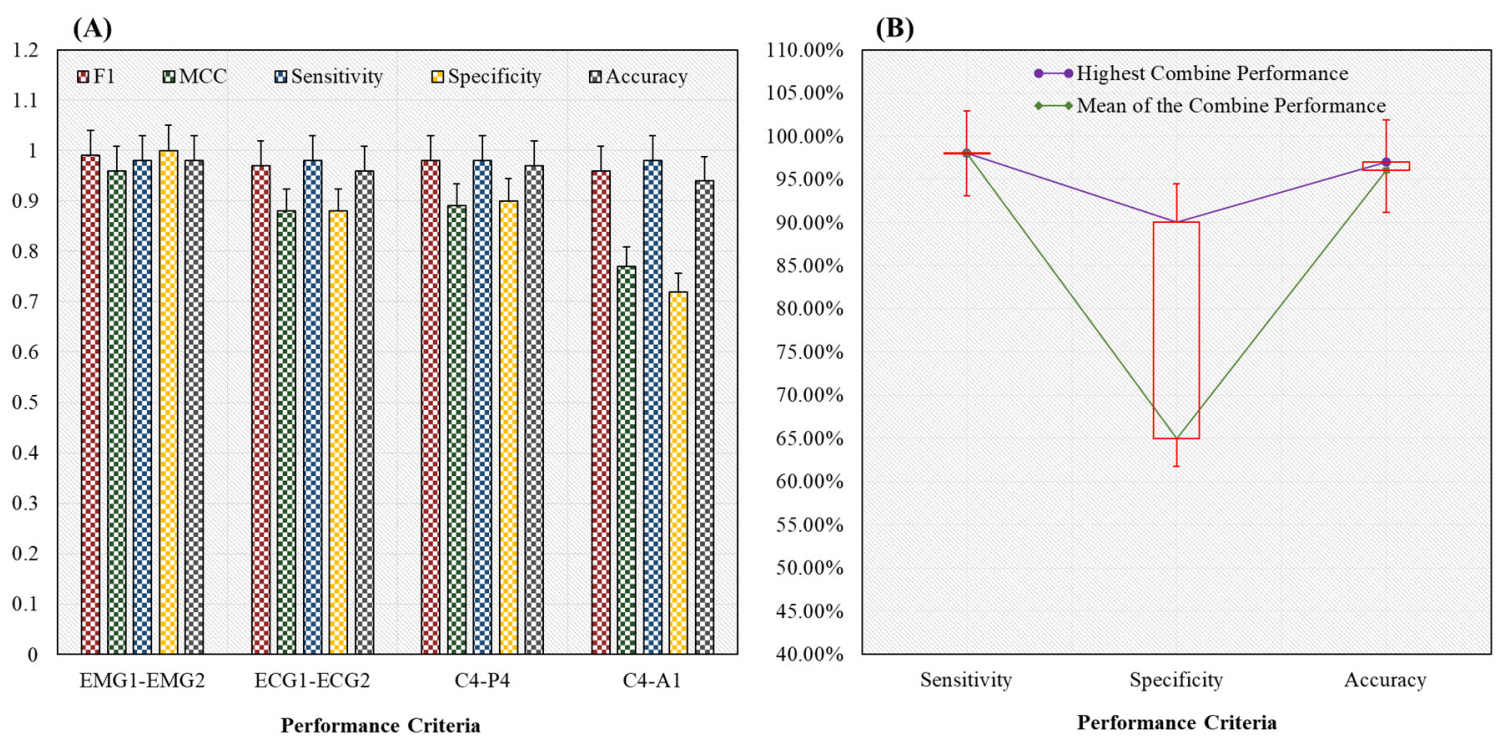

Figure 9. Performance analysis for the combination of subjects (bruxism and healthy) and sleep stages ( $w$ and REM) classification using the novel 20-fold HML classifier. (A) Comparison between all channels such as EMG1-EMG2, ECG1, ECG2, C4-P4, and C4-A1 channels of the sleep stages classification. In addition, (B) highest and mean performance of the combined classification. The C4-P4 channel of the EEG signal has the highest accuracy $(97 \%)$ in the combined classification.

\subsection{Comparison with the Previous and Proposed Sleep Disorders and Sleep Stage Detection Methods}

Performances of the subjects (healthy and bruxism) classification on the EEG (C4-A1 channel) signal in terms of sensitivity, specificity, and accuracy were found to be $0.95,0.92$, and 0.94 , respectively. The performance of the sleep stages ( $w$ and REM) classification on the ECG (ECG1-ECG2 channel) signal in terms of sensitivity, specificity, and accuracy were found to be $1,0.86$, and 0.95 , respectively. Additionally, the performance of the combined (subjects and sleep stages) classification on the EEG (C4-P4 channel) signal in terms of sensitivity, specificity, and accuracy were found to be $0.98,0.90$, and 0.97 , respectively. The C4-P4 channel of the combined classification has better performance than the others. Previously, bruxism detection methods were limited, so we compared with sleep disorder and sleep stage detection methods. Patients of bruxism are recognized through various diagnosis methods involving questionnaires, clinical examinations, and various appliances. The questionnaires involve the patient history of tooth mobility and tooth wear, muscle pain, hypersensitivity of teeth and masticatory muscle discomfort, fatigue, or pain [62]. Clinically, Ekfeldt et al. [63] detected a bruxism patient through intraoral and extraoral examination. Takeuchi et al. [64] suggested that bruxism is more accurately measured extraorally through masticatory muscle EMG recordings and polysomnography. We compare our proposed method with previously selected sleep disorders and sleep stage methods in terms of author, publication year, subject detection, signal, classifier, sensitivity, specificity, and accuracy, as mentioned in Table 5. The data from sleep disorders and sleep stage detection methods include EEG, EMG, and ECG signals. The classifiers involve decision tree (DT), KNN, SVM RBF kernel, threshold, and the novel HML classifier. Compared with them, our method shows a better performance in detecting bruxism (Figure 10A). Additionally, the proposed HML classifier is also compared with some existing hybrid classifiers, which are reported in Table 6. Our proposed HML classifier achieves satisfactory performance in detecting bruxism (Figure 10B). 
Table 5. Comparison of the proposed method with the existing methods.

\begin{tabular}{|c|c|c|c|c|c|c|c|}
\hline Reference & Year & Disease & Signal & Classifier & Sen $(\%)$ & Spe $(\%)$ & Acc $(\%)$ \\
\hline Heyat et al. [40] & 2019 & Bruxism & EEG & DT & 89 & 78 & 81 \\
\hline Lai et al. [19] & 2019 & Bruxism & EMG & DT & 94 & 92 & 93 \\
\hline Bhattacharjee et al. [65] & 2019 & SA & EEG & KNN & 98 & 83 & 91 \\
\hline Zarei et al. [66] & 2019 & OSA & ECG & SVM RBF Kernel & 94 & 94 & 94 \\
\hline Dong et al. [67] & 2018 & OSA & ECG & Threshold & 88 & 90 & 90 \\
\hline Kassiri et al. [68] & 2017 & Sleep Stage & EEG, EMG & Threshold & 81 & 93 & 81 \\
\hline Kohtoh et al. [69] & 2008 & Sleep Stage & EEG, EMG & Threshold & 71 & 96 & 84 \\
\hline Louis et al. [70] & 2004 & Sleep Stage & EEG, EMG & Threshold & 66 & 84 & 82 \\
\hline \multirow{3}{*}{ Proposed } & \multirow{2}{*}{\multicolumn{2}{|c|}{$\begin{array}{c}\text { Subjects } \\
\text { (Bruxism and Healthy) } \\
\text { Sleep Stages } \\
\text { (w and REM) }\end{array}$}} & $\begin{array}{l}\text { EEG } \\
(\mathrm{C} 4-\mathrm{A} 1)\end{array}$ & \multirow{3}{*}{ HML } & 95 & 92 & 94 \\
\hline & & & $\begin{array}{c}\text { ECG } \\
\text { (ECG1-ECG2) }\end{array}$ & & 100 & 86 & 95 \\
\hline & \multicolumn{2}{|c|}{$\begin{array}{c}\text { Combine } \\
\text { (Subjects and Sleep Stages) }\end{array}$} & $\begin{array}{l}\text { EEG } \\
(\mathrm{C} 4-\mathrm{P} 4)\end{array}$ & & 98 & 90 & 97 \\
\hline
\end{tabular}

SA: sleep apnea; OSA: obstructive sleep apnea; DT: decision tree. Bold is the best model.
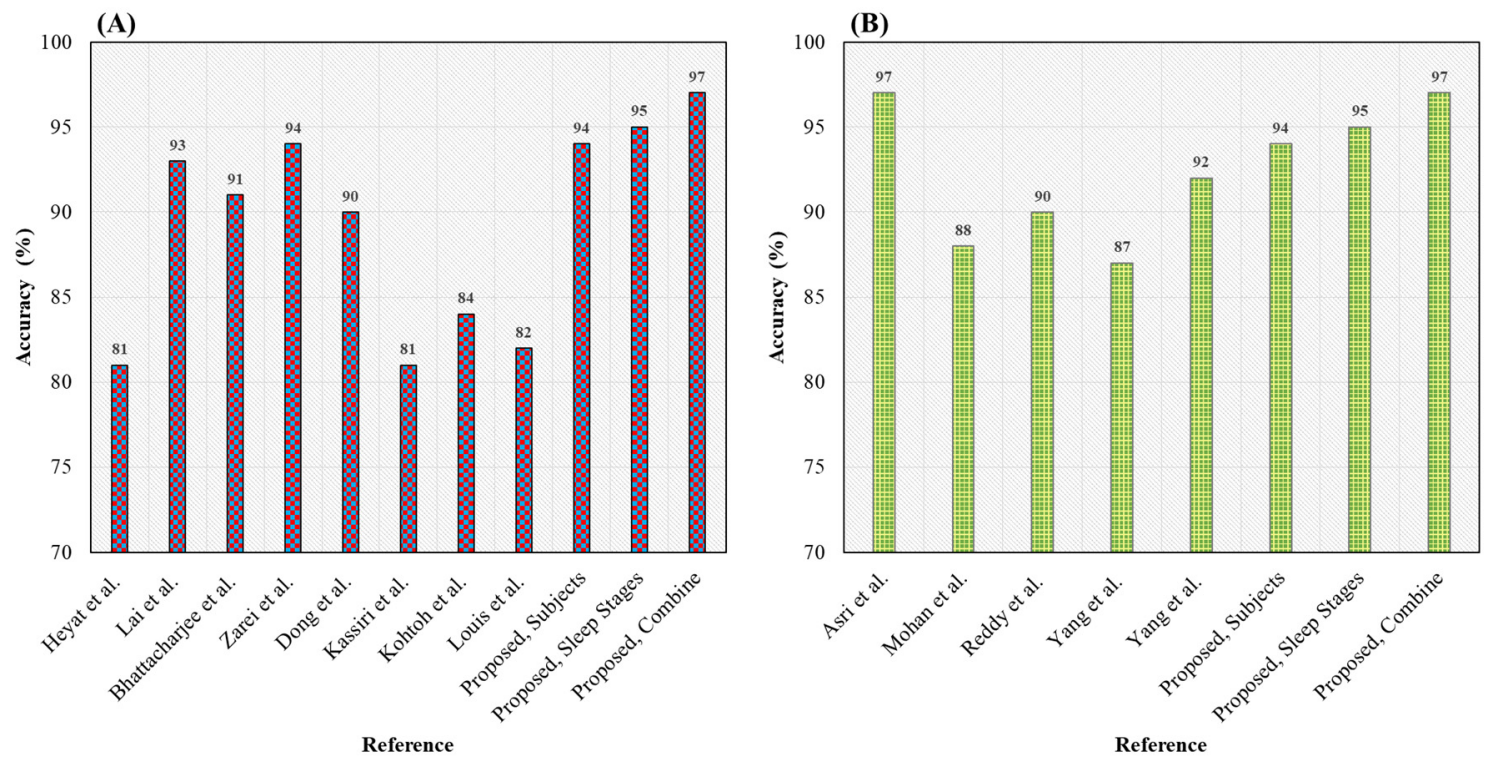

Figure 10. A comparison of the (A) proposed method with the existing methods and (B) proposed HML classifier with the existing hybrid classifiers.

Table 6. Comparison of the proposed HML classifier with the existing hybrid classifiers.

\begin{tabular}{cccc}
\hline Reference & Year & Disease & Acc (\%) \\
\hline Asri et al. [71] & 2020 & Cancer & 97 \\
Mohan et al. [72] & 2019 & Heart & 88 \\
Reddy et al. [73] & 2019 & Heart & 90 \\
Yang et al. [74] & 2010 & Schizophrenia & 87 \\
Yang et al. [75] & 2007 & Cushing & 92 \\
\hline & & & 94 \\
Proposed & & Bruxism & 95 \\
& & & $\mathbf{9 7}$ \\
\hline
\end{tabular}

Bold is the best model.

\subsection{Application and Limitation of the Proposed System}

The proposed work showed an application for the detection of bruxism disorder and sleep stages using ECG1-ECG2, EMG1-EMG2, C4-P4, and C4-A1 channels. This work would provide a more 
effective and accurate detection system of bruxism and sleep stages for medical application. The most important application of the present research is to diagnose people with a mental health condition in a fast and accurate manner. This system also helps to find the problem during the sleep stage.

The present work has some limitations in that the proposed data from the physionet website were old and small for the evaluation. Further work could be required for a large number of real-time data to analyze the present work for higher accuracy. Secondly, the proposed physiological signals, including ECG, EEG, and EMG, did not cover all sleep recordings. Moreover, we used two sleep stages, including $w$ and REM. In the future, we will use different signals and different sleep stages for the detection of bruxism sleep disorders.

\section{Conclusions}

Bruxism is a sleep disorder in which people clinch, grind, and gnash their teeth during sleep. In this proposed study, we have developed a novel HML classifier to detect bruxism. The results show that the HML classifier can effectively discriminate subjects, sleep stages, and these two combined (subjects and sleep stages) with 94, 95, and 97\% accuracy, respectively. In our knowledge, this method (HML classifier) is used for the first time to detect bruxism. We summarized that the EEG (C4-P4 channel) signal of the HML classifier could be utilized in bruxism detection. This method can achieve an effective result with PSD with potential application for sleep disorder and sleep stage detection. The upcoming study will quickly detect sleep disorders with higher accuracy.

Author Contributions: M.B.B.H.: data collection, research design, simulation, and writing; F.A.: research design and writing; A.K.: simulation; A.N.: proofreading and formatting; B.B.: review and rewriting; Y.: research design; S.J.A.: proofreading; D.L.: proofreading. All authors have read and agreed to the published version of the manuscript.

Funding: This work was supported by the Robotics and Internet-of-Things Laboratory of Prince Sultan University, Saudi Arabia, and by the National Natural Science Foundation of China (61771100).

Conflicts of Interest: The authors declare no conflict of interest.

\section{References}

1. Crowley, K. Sleep and sleep disorders in older adults. Neuropsychol. Rev. 2011, 21, 41-53. [CrossRef] [PubMed]

2. Menghini, L.; Alschuler, V.; Claudatos, S.; Goldstone, A.; Baker, F.; Cellini, N.; Colrain, I.M.; de Zambotti, M. 1193 Accuracy of a Commercial Wearable in Detecting Sleep Stages Compared to Polysomnography in Adults: Considering Sleep Classification Methods and Effects of Evening Alcohol Consumption. Sleep 2020, 43, A456-A457. [CrossRef]

3. Rechtschaffen, A.; Kales, A. A Manual of Standardized Terminology, Techniques and Scoring System for Sleep Stages of Human Subjects (Part 2); National Institutes of Health (U.S.): Bethesda, MD, USA, 1968.

4. Grigg-Damberger, M.M. The AASM scoring manual four years later. J. Clin. Sleep Med. 2012, 8, 323-332. [CrossRef] [PubMed]

5. Anderer, P.; Moreau, A.; Woertz, M.; Ross, M.; Gruber, G.; Parapatics, S.; Loretz, E.; Heller, E.; Schmidt, A.; Boeck, M.; et al. Computer-assisted sleep classification according to the standard of the American Academy of sleep medicine: Validation study of the AASM version of the Somnolyzer $24 \times 7$. Neuropsychobiology 2010, 62, 250-264. [CrossRef] [PubMed]

6. Wells, M.E.; Vaughn, B.V. Poor sleep challenging the health of a nation. Neurodiagn. J. 2012, 52, $233-249$. [CrossRef] [PubMed]

7. Abdulghani, H.M.; Alrowais, N.A.; Bin-Saad, N.S.; Al-Subaie, N.M.; Haji, A.M.; Alhaqwi, A.I. Sleep disorder among medical students: Relationship to their academic performance. Med. Teach. 2012, 34, S37-S41. [CrossRef] [PubMed]

8. Hasan, Y.M.; Bin Heyat, M.B.; Siddiqui, M.M.; Azad, S.; Akhtar, F. An Overview of Sleep and Stages of Sleep. Int. J. Adv. Res. Comput. Commun. Eng. 2015, 4, 505-507.

9. Asif, N.; Iqbal, R.; Nazir, C.F. Human immune system during sleep. Am. J. Clin. Exp. Immunol. 2017, 6, 92.

10. Barnes, C.M.; Schaubroeck, J.; Huth, M.; Ghumman, S. Lack of sleep and unethical conduct. Organ. Behav. Hum. Decis. Process. 2011, 115, 169-180. [CrossRef] 
11. Heyat, M.B.B.; Lai, D.; Akhtar, F.; Hayat, M.A.B.; Azad, S.; Azad, S.; Azad, S. Bruxism Detection Using Single-Channel C4-A1 on Human Sleep S2 Stage Recording. In Intelligent Data Analysis: From Data Gathering to Data Comprehension, 1st ed.; Gupta, D., Bhattacharyya, S., Khanna, A., Eds.; John Wiley \& Sons: Hoboken, NJ, USA, 2020; pp. 347-367.

12. Hillman, D.R.; Lack, L.C. Public health implications of sleep loss: The community burden. Med. J. Aust. 2013, 199, S7-S10. [CrossRef]

13. Liu, X.; Ma, Y.; Wang, Y.; Jiang, Q.; Rao, X.; Lu, X.; Teng, H. Brief report: An epidemiologic survey of the prevalence of sleep disorders among children 2 to 12 years old in Beijing, China. Pediatrics 2005, 115, $266-268$. [CrossRef]

14. Casson, A.J.; Smith, S.; Duncan, J.S.; Rodriguez-Villegas, E. Wearable EEG: What is it, why is it needed and what does it entail? In Proceedings of the 2008 30th Annual International Conference of the IEEE Engineering in Medicine and Biology Society, Vancouver, BC, Canada, 20-24 August 2008. [CrossRef]

15. Panda, S.; Taly, A.B.; Sinha, S.; Gururaj, G.; Girish, N.; Nagaraja, D. Sleep-related disorders among a healthy population in South India. Neurol. India 2012, 60, 68. [CrossRef]

16. Monma, T.; Ando, A.; Asanuma, T.; Yoshitake, Y.; Yoshida, G.; Miyazawa, T.; Ebine, N.; Takeda, S.; Omi, N.; Satoh, M.; et al. Sleep disorder risk factors among student athletes. Sleep Med. 2018, 44, 76-81. [CrossRef]

17. Melo, G.; Duarte, J.; Pauletto, P.; Porporatti, A.L.; Stuginski-Barbosa, J.; Winocur, E.; Flores-Mir, C.; De Luca Canto, G. Bruxism: An umbrella review of systematic reviews. J. Oral Rehabil. 2019, 46, 666-690. [CrossRef] [PubMed]

18. Lal, S.J.; Weber, K.K. Bruxism Management. In StatPearls; StatPearls Publishing: Treasure Island, FL, USA, 2018.

19. Heyat, M.B.B.; Lai, D.; Khan, F.I.; Zhang, Y. Sleep Bruxism Detection Using Decision Tree Method by the Combination of C4-P4 and C4-A1 Channels of Scalp EEG. IEEE Access 2019, 7, 102542-102553. [CrossRef]

20. Lai, D.; Zhang, X.; Zhang, Y.; Heyat, M.B.B. Convolutional Neural Network Based Detection of Atrial Fibrillation Combing R-R intervals and F-wave Frequency Spectrum. In Proceedings of the 2019 41st Annual International Conference of the IEEE Engineering in Medicine and Biology Society (EMBC), Berlin, Germany, 23-27 July 2019.

21. Lai, D.; Zhang, Y.; Zhang, X.; Su, Y.; Heyat, M.B.B. An Automated Strategy for Early Risk Identification of Sudden Cardiac Death by Using Machine Learning Approach on Measurable Arrhythmic Risk Markers. IEEE Access 2019, 7, 94701-94716. [CrossRef]

22. Heyat, M.B.B.; Hasan, Y.M.; Siddiqui, M.M. EEG signals and wireless transfer of EEG Signals. Int. J. Adv. Res. Comput. Commun. Eng. 2015, 4, 10-12.

23. Heyat, M.B.B.; Siddiqui, M.M. Recording of EEG, ECG, EMG Signal. Int. J. Adv Res. Comput. Sci. Softw. Eng. 2015, 5, 813-815.

24. Imtiaz, S.A.; Rodriguez-Villegas, E. A Low Computational Cost Algorithm for REM Sleep Detection Using Single Channel EEG. Ann. Biomed. Eng. 2014, 42, 2344-2359. [CrossRef] [PubMed]

25. Guillot, M.; Jungo, S.; Maniere, A.; Laplanche, O.; Tillier, Y.; Ehrmann, E. Diagnosis and management of bruxism: Evaluation of clinical practices in France. Cranio 2019, 1-12. [CrossRef] [PubMed]

26. Saczuk, K.; Lapinska, B.; Wilmont, P.; Pawlak, L.; Lukomska-Szymanska, M. The Bruxoff Device as a Screening Method for Sleep Bruxism in Dental Practice. J. Clin. Med. 2019, 8, 930. [CrossRef] [PubMed]

27. Maeda, M.; Yamaguchi, T.; Mikami, S.; Yachida, W.; Saito, T.; Sakuma, T.; Nakamura, H.; Saito, M.; Mizuno, M.; Yamada, K.; et al. Quantitative analyses of jaw-opening muscle activity during the active phase of jaw-closing muscles in sleep bruxism. J. Sleep Res. 2019, e12922. [CrossRef] [PubMed]

28. Miettinen, T.; Myllymaa, K.; Muraja-Murro, A.; Westeren-Punnonen, S.; Hukkanen, T.; Töyräs, J.; Lappalainen, R.; Mervaala, E.; Sipilä, K.; Myllymaa, S. Screen-printed ambulatory electrode set enables accurate diagnostics of sleep bruxism. J. Sleep Res. 2018, 27, 103-112. [CrossRef] [PubMed]

29. Ruhland, J.L.; Jeutter, D.C.; Ackmann, J.J.; Hoge, H.W.; Jodat, R.W. Acquisition and analysis of electromyograms of the human masseter muscle. In Proceedings of the Annual International Conference of the IEEE Engineering in Medicine and Biology Society, New Orleans, LA, USA, 4-7 November 1988. [CrossRef]

30. Martínez, A.; Barrientos, A.; Díaz, A.; Lafont, P.; Colorado, J.; Castedo, P.L.; González, R. Polymeric piezoelectric sensors and remote communication for detection of Bruxism. In Proceedings of the 2010 IEEE International Conference on Industrial Technology, Vina del Mar, Chile, 14-17 March 2010. [CrossRef] 
31. Kostka, P.S.; Tkacz, E.J. Multi-sources data analysis with sympatho-vagal balance estimation toward early bruxism episodes detection. In Proceedings of the 2015 37th Annual international conference of the IEEE engineering in medicine and biology society (EMBC), Milan, Italy, 25-29 August 2015. [CrossRef]

32. Jirakittayakorn, N.; Wongsawat, Y. An EMG instrument designed for bruxism detection on masseter muscle. In Proceedings of the 7th 2014 Biomedical Engineering International Conference, Fukuoka, Japan, 26-28 November 2014. [CrossRef]

33. Bb, H.; Akhtar, F.; Mehdi, A.; Azad, S.; Azad, S.; Azad, S. Normalized Power are used in the Diagnosis of Insomnia Medical Sleep Syndrome through EMG1-EMG2 Channel. Austin J. Sleep Disord. 2017, 4, $2-4$.

34. Heyat, M.B.B.; Akhtar, F.; Ammar, M.; Hayat, B.; Azad, S. Power Spectral Density are used in the Investigation of insomnia neurological disorder. In Proceedings of the XL- Pre Congress Symposium, organized by Indian Academy of Social Sciences, State Takmeel-ut-Tib-College \& Hospital, and King George Medical College \& Hospital, Lucknow, UP, India, October 2016; pp. 45-50.

35. Heyat, M.B.B.; Akhtar, F.; Sikandar, M.; Siddiqui, H.; Azad, S. An Overview of Dalk Therapy and Treatment of Insomnia in Dalk Therapy Abstract- Treatment of Insomnia in Dalk Therapy. In Proceedings of the National Seminar on Research Methodology in Ilaj-Bit-Tadbeer, organized by State Takmeel-ut-Tib-College \& Hospital, Lucknow, India, 10-11 October 2015.

36. Bin Heyat, B.; Akhtar, F.; Singh, S.K.; Siddiqui, M.M. Hamming Window are used in the Prognostic of Insomnia. In Proceedings of the International Seminar Present Scenario Future Prospectives Res. Eng. Sci. (ISPSFPRES), organized by Integral University, Lucknow, UP, India, January 2017; pp. 65-71.

37. Heyat, M.B.B. Insomnia: Medical Sleep Disorder E Diagnosis, 1st ed.; Anchor Academic Publishing: Hamburg, Germany, 2016.

38. Heyat, M.B.B.; Akhtar, F.; Azad, S. Comparative Analysis of Original Wave and Filtered Wave of EEG signal Used in the Prognostic of Bruxism medical Sleep syndrome. Int. J. Trend Sci. Res. Dev. 2016, 1,7-9. [CrossRef]

39. Heyat, M.B.B.; Lai, D.; Akhtar, F.; Hayat, M.A.B. Short Time Frequency Analysis of Theta Activity for the Diagnosis of Bruxism on EEG Sleep. In Advanced Computational Intelligence Techniques for Virtual Reality in Healthcare Studies in Computational Intelligence; Gupta, K.D., Hassanien, A., Eds.; Springer: Berlin/Heidelberg, Germany, 2020; pp. 63-83.

40. Lai, D.; Heyat, M.B.B.; Khan, F.I.; Zhang, Y. Prognosis of Sleep Bruxism Using Power Spectral Density Approach Applied on EEG Signal of Both EMG1-EMG2 and ECG1-ECG2 Channels. IEEE Access 2019, 7, 82553-82562. [CrossRef]

41. Rahman, T.; Farook, O.; Bin Heyat, B.; Siddiqui, M.M. An Overview of Narcolepsy. Int. Adv. Res. J. Sci. Eng. Technol. 2016, 3, 2393-2395.

42. Farooq, O.; Rahman, T.; Bin Heyat, B.; Siddiqui, M.M.; Akhtar, F. An Overview of NFLE. Int. J. Innov. Res. Electr. Electron. Instrum. Control. Eng. 2016, 4, 209-211.

43. Shahin, M.; Ahmed, B.; Hamida, S.T.B.; Mulaffer, F.L.; Glos, M.; Penzel, T. Deep Learning and Insomnia: Assisting Clinicians with Their Diagnosis. IEEE J. Biomed. Heal. Inform. 2017, 21, 1546-1553. [CrossRef]

44. Mulaffer, L.; Shahin, M.; Glos, M.; Penzel, T.; Ahmed, B. Comparing two insomnia detection models of clinical diagnosis techniques. In Proceedings of the 39th Annual International Conference of the IEEE Engineering in Medicine and Biology Society (EMBC), Jeju Island, Korea, 11-15 July 2017. [CrossRef]

45. Goldberger, A.L.; Amaral, L.A.; Glass, L.; Hausdorff, J.M.; Ivanov, P.C.; Mark, R.G.; Mietus, J.E.; Moody, G.B.; Peng, C.-K.; Eugene Stanley, H.; et al. PhysioBank, PhysioToolkit, and PhysioNet. Circulation 2000, 101, e215-e220. [CrossRef]

46. Costa, M.; Moody, G.B.; Henry, I.; Goldberger, A.L. PhysioNet: An NIH Research Resource for Complex Signals. J. Electrocardiol. 2003, 36, 139-144. [CrossRef] [PubMed]

47. Welch, P.D. Welch_1967_Modified_Periodogram_Method. Trans. Audio Electroacoust. 1967, 15, 70-73. [CrossRef]

48. Siddiqui, M.M.; Srivastava, G.; Saeed, S.H. Diagnosis of insomnia sleep disorder using short time frequency analysis of PSD approach applied on EEG signal using channel ROC-LOC. Sleep Sci. 2016, 9, 186-191. [CrossRef] [PubMed]

49. Barbé, K.; Pintelon, R.; Schoukens, J. Welch method revisited: Nonparametric power spectrum estimation via circular overlap. IEEE Trans. Signal. Process. 2010, 58, 553-565. [CrossRef]

50. Liu, D.; Pang, Z.; Lloyd, S.R. A neural network method for detection of obstructive sleep apnea and narcolepsy based on pupil size and EEG. IEEE Trans. Neural Netw. 2008, 19, 308-318. [CrossRef] 
51. Rahi, P.K.; Mehra, R. Analysis of Power Spectrum Estimation Using Welch Method for Various Window Techniques. Int. J. Emerg. Technol. Eng. 2014, 2, 106-109.

52. Xiang, C.; Yong, P.C.; Meng, L.S. Design of multiple-level hybrid classifier for intrusion detection system using Bayesian clustering and decision trees. Pattern Recognit. Lett. 2008, 29, 918-924. [CrossRef]

53. Bezdek, J.C.; Reichherzer, T.R.; Lim, G.S.; Attikiouzel, Y. Multiple-prototype classifier design. IEEE Trans. Syst. Man Cybern. Part C Appl. Rev. 1998, 28, 67-79. [CrossRef]

54. Chen, S.; Shen, B.; Wang, X.; Yoo, S.J. A strong machine learning classifier and decision stumps based hybrid adaboost classification algorithm for cognitive radios. Sensors 2019, 19, 5077. [CrossRef]

55. Rawat, K.S.; Malhan, I.V. A Hybrid Classification Method Based on Machine Learning Classifiers to Predict Performance in Educational Data Mining. In Lecture Notes in Networks and Systems; Springer: Singapore, 2019.

56. Miškovic, V. Machine Learning of Hybrid Classification Models for Decision Support. Sinteza 2014 Impact Internet Bus. Act. Serbia Worldw. 2014, 2014, 318-324. [CrossRef]

57. Chen, T.; Sun, J.; Lin, H.; Liu, Y. Hybrid. Machine Learning Models of Classifying Residential Requests for Smart Dispatching; Springer: Singapore, 2019. [CrossRef]

58. Längkvist, M.; Karlsson, L.; Loutfi, A. Sleep Stage Classification Using Unsupervised Feature Learning. Adv. Artif. Neural Syst. 2012, 2012, 107046. [CrossRef]

59. Boe, A.J.; Koch, L.L.M.; O’Brien, M.K.; Shawen, N.; Rogers, J.A.; Lieber, R.L.; Reid, K.J.; Zee, P.C.; Jayaraman, A. Automating sleep stage classification using wireless, wearable sensors. NPJ Digit. Med. 2019, 2, 1-9. [CrossRef] [PubMed]

60. Bajaj, V.; Pachori, R.B. Automatic classification of sleep stages based on the time-frequency image of EEG signals. Comput. Methods Prog. Biomed. 2013, 112, 320-328. [CrossRef] [PubMed]

61. Mitsukura, Y.; Fukunaga, K.; Yasui, M.; Mimura, M. Sleep stage detection using only heart rate. Health Inform. J. 2019, 26, 376-387. [CrossRef]

62. Pintado, M.R.; Anderson, G.C.; DeLong, R.; Douglas, W.H. Variation in tooth wear in young adults over a two-year period. J. Prosthet. Dent. 1997, 77, 313-320. [CrossRef]

63. Ekfeldt, A.; Hugoson, A.; Bergendal, T.; Helkimo, M. An individual tooth wear index and an analysis of factors correlated to incisal and occlusal wear in an adult swedish population. Acta Odontol. Scand. 1990, 48, 343-349. [CrossRef]

64. Takeuchi, H.; Ikeda, T.; Clark, G.T. A piezoelectric film-based intrasplint detection method for bruxism. J. Prosthet. Dent. 2001, 86, 195-202. [CrossRef]

65. Bhattacharjee, A.; Saha, S.; Fattah, S.A.; Zhu, W.P.; Ahmad, M.O. Sleep apnea detection based on rician modeling of feature variation in multiband EEG signal. IEEE J. Biomed. Heal. Inform. 2018, 23, 1066-10174. [CrossRef]

66. Zarei, A.; Asl, B.M. Automatic Detection of Obstructive Sleep Apnea Using Wavelet Transform and Entropy-Based Features from Single-Lead ECG Signal. IEEE J. Biomed. Heal. Inform. 2018, 23, 1011-1021. [CrossRef]

67. Dong, Z.; Li, X.; Chen, W. Frequency Network Analysis of Heart Rate Variability for Obstructive Apnea Patient Detection. IEEE J. Biomed. Heal. Inform. 2017, 22, 1895-1905. [CrossRef]

68. Kassiri, H.; Chemparathy, A.; Salam, M.T.; Boyce, R.; Adamantidis, A.; Genov, R. Electronic Sleep Stage Classifiers: A Survey and VLSI Design Methodology. IEEE Trans. Biomed. Circ. Syst. 2017, 11, 177-188. [CrossRef]

69. Kohtoh, S.; Taguchi, Y.; Matsumoto, N.; Wada, M.; Huang, Z.L.; Urade, Y. Algorithm for sleep scoring in experimental animals based on fast Fourier transform power spectrum analysis of the electroencephalogram. Sleep Biol. Rhythms 2008, 6, 163-171. [CrossRef]

70. Louis, R.P.; Lee, J.; Stephenson, R. Design and validation of a computer-based sleep-scoring algorithm. J. Neurosci. Methods 2004, 133, 71-80. [CrossRef]

71. Asri, H.; Mousannif, H.; Al Moatassim, H. A Hybrid. Data Mining Classifier for Breast Cancer Prediction; Springer: Cham, Swizerland, 2020. [CrossRef]

72. Mohan, S.; Thirumalai, C.; Srivastava, G. Effective heart disease prediction using hybrid machine learning techniques. IEEE Access 2019, 7, 81542-81554. [CrossRef]

73. Reddy, G.T.M.; Reddy, P.K.; Lakshmanna, K.; Rajput, D.S.; Kaluri, R.; Srivastava, G. Hybrid genetic algorithm and a fuzzy logic classifier for heart disease diagnosis. Evol. Intell. 2020, 13, 185-196. [CrossRef] 
74. Yang, H.; Liu, J.; Sui, J.; Pearlson, G.; Calhoun, V.D. A hybrid machine learning method for fusing fMRI and genetic data: Combining both improves classification of schizophrenia. Front. Hum. Neurosci. 2010, 4, 192. [CrossRef] [PubMed]

75. Yang, J.Y.; Yang, M.Q.; Luo, Z.; Ma, Y.; Li, J.; Deng, Y.; Huang, X. A hybrid machine learning-based method for classifying the Cushing's Syndrome with comorbid adrenocortical lesions. BMC Genom. 2008, 9, S23. [CrossRef]

Publisher's Note: MDPI stays neutral with regard to jurisdictional claims in published maps and institutional affiliations.

(C) 2020 by the authors. Licensee MDPI, Basel, Switzerland. This article is an open access article distributed under the terms and conditions of the Creative Commons Attribution (CC BY) license (http://creativecommons.org/licenses/by/4.0/). 